\title{
Löfgren's Syndrome: Acute Variant of Sarcoidosis
}

\author{
M. AZZZUL HAQUE'르. M. GOLAM MOSTAFA², ARM SAIFUDDIN EKRAM ${ }^{3}$, AKM SHOHIDUR RAHMANTARAFDAR ${ }^{4}$
}

\begin{abstract}
Löfgren's syndrome is an acute form of sarcoidosis that is characterized by erythema nodosum, bilateral hilar lymphadenopathy accompanied by arthritis or arthralgia. We are reporting a 32 year old male with Löfgren's syndrome and then the literature is reviewed.
\end{abstract}

Keyword: Löfgren's syndrome, sarcoidosis, erythema nodosum, hilar lymphadenopathy, Bangladesh

\section{Introduction}

Sarcoidosis is an enigmatic chronic multisystem inflammatory condition of obscure etiology, primarily affecting young adults aged between 20 and 40 years. ${ }^{1,2}$ The lungs are the most frequently affected organ ( $>90 \%)$, but the skin, eyes, liver, and lymph nodes are commonly involved. ${ }^{3,4}$ The modern history of sarcoidosis goes back to 1899, when the pioneering Norwegian dermatologist Caesar Boeck coined the term to describe skin nodules characterized by compact, sharply defined foci of "epithelioid cells with large pale nuclei and also a few giant cells." Thinking this resembled sarcoma, he called the condition "multiple benign sarcoid of the skin. ${ }^{5}$ There are two eponymous syndromes associated with sarcoidosis. Heerfordt syndrome is the symptom triad of uveitis, parotid gland enlargement and cranial nerve paresis and was described by Danish ophthalmologist C. F. Heerfordt in $1909 .{ }^{6}$ Löfgren's syndrome is a form of acute-type sarcoidosis that is characterized by erythema nodosum (EN), bilateral hilar lymphadenopathy (BHL) accompanied by arthritis or arthralgia. ${ }^{7}$ It was first recognized by Sven Lofgren in 1953 in a series of 113 patients. ${ }^{8,9}$ Sarcoidosis affects people of all racial and ethnic groups and occurs at all ages, with the incidence peaking at 20 to 39 year. ${ }^{10}$ The highest annual incidence of sarcoidosis has been observed in northern European countries (5 to 40 cases per 100,000 people). ${ }^{11}$ The adjusted annual incidence among black Americans is roughly three times that among white Americans. ${ }^{10}$

In Bangladesh, there is not much statistical data regarding the prevalence or pattern of sarcoidosis. Because of rarity of this disease and inexperience of some cytopathologists, many cases clinically consistent with sarcoidosis are falsely labeled as tuberculosis. We believe that sarcoidosis is more prevalent in Bangladesh than currently known. We are reporting an adult male with Löfgren's syndrome, an acute variant of sarcoidosis, affecting skin, musculoskeletal, ocular and respiratory systems.

\section{Case Report}

A 32 year old male presented to Department of Medicine, Rajshahi Medical College with the complaints of pain in ankles, knees, wrists and elbows, redness and watering from both eyes and vague chest discomfort for 4 weeks. His joint pain was severe enough to hamper his daily activities. He did not have fever, cough, night sweats, weight loss, breathlessness, syncope, headaches, nausea, vomiting, abdominal pain, diarrhea, dysuria, and skin rash. He does not give any past history of tuberculosis or recent contact with patients of tuberculosis. Treatment with NSAIDs did not help. On examination he was not anemic, lymph nodes were not palpable, and salivary glands were not enlarged. Erythema nodosum was present in both shins. His ankle, knee, wrist and elbow joints were tender on palpation. He had mild redness in both eyes. Examination of respiratory, gastrointestinal, CVS and nervous system were unremarkable. His complete blood count was normal, ESR was 40. RA test was negative, so was the antinuclear antibody. Slit lamp examination of the eyes revealed bilateral anterior uveitis, but his visual acuity was noted to be normal in both eyes. CXR showed bilateral hilar lymphadenopathy (Fig. 1). USG of whole abdomen was normal. Mantoux test showed no induration. CT scan of chest demonstrated bilateral hilar lymphadenopathy (Fig.-2), pulmonary parenchyma was normal. CT guided FNAC of hilar lymph node was reported as: non-caseating granulomatous inflammation compatible with sarcoidosis (Fig.-3, 4). Serum calcium was $9.2 \mathrm{mg} / \mathrm{dL}$. Routine urine examination, liver and renal functions and ECG were normal.

1. Assistant Professor, Department of Medicine, Rajshahi Medical College, Rajshahi

2. Professor, Histopathology, National Institute of Cancer Research and Hospital, Dhaka

3. Professor, Department of Medicine, Rajshahi Medical College, Rajshahi

4. Associate Professor, Department of Ophthalmology, Rajshahi Medical College, Rajshahi

Correspondence: Dr. M. Azizul Haque, Assistant Professor, Department of Medicine, Rajshahi Medical College, Email:drazadbd@gmail.com 

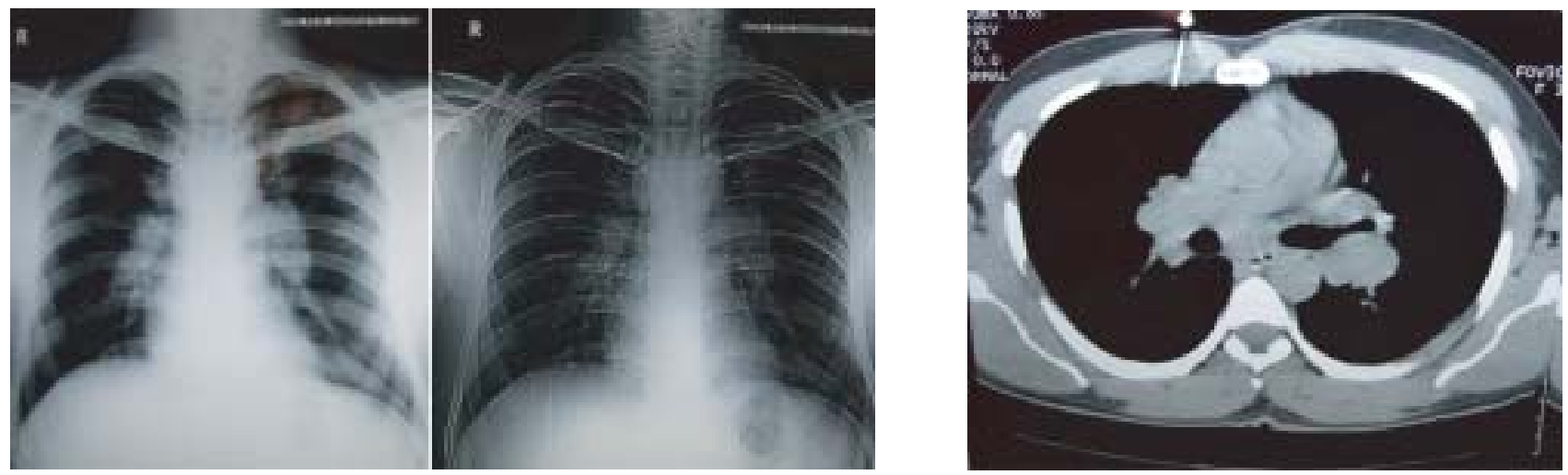

Fig.-1 \& 2: CXR and CT scan chest showing bilateral hilar lymphadenopathy

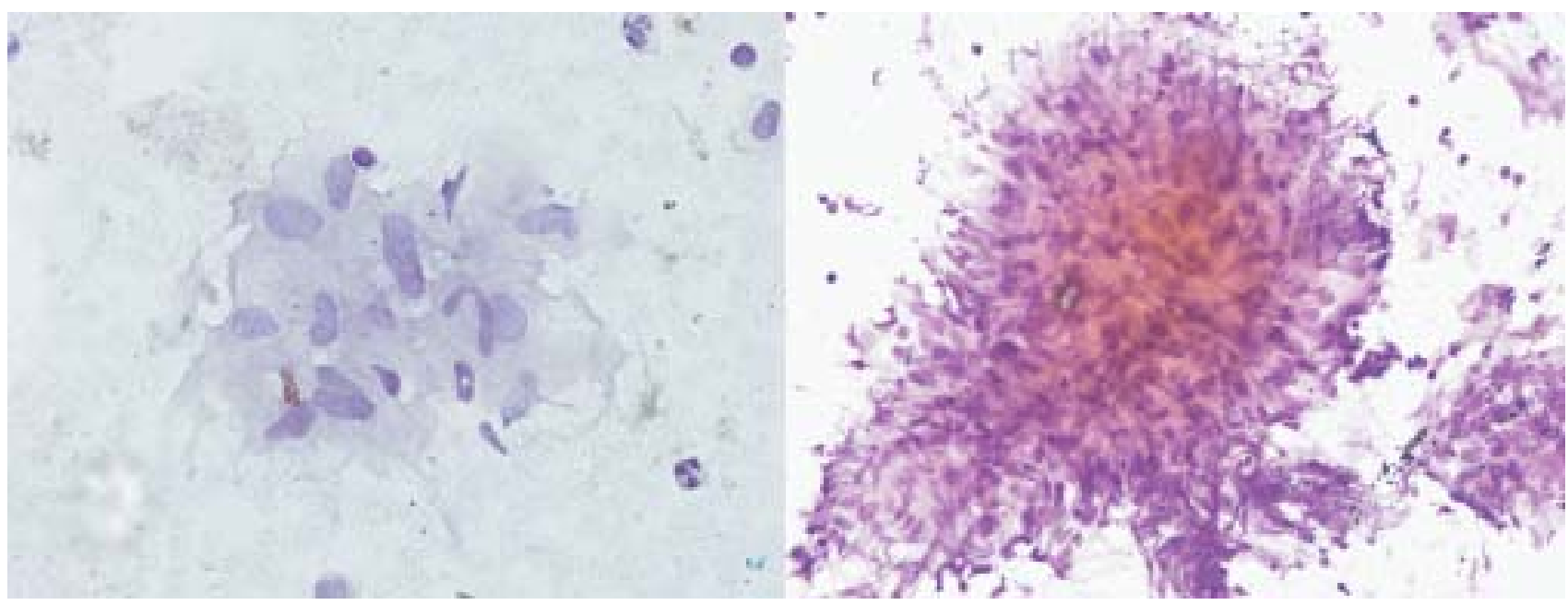

Fig.-3 \& 4: Photomicrograph showing non-caseating granuloma

Our patient was diagnosed as a case of Löfgren's syndrome based on his classical clinical picture: BHL, EN, and arthritis and it was supported by presence of non-caseating granuloma on FNAC of hilar lymph node. Prednisolone was started at a dose of $40 \mathrm{mg} /$ day; patient became asymptomatic after 15 days. Prednisolone was being tapered. Bilateral hilar lymphadenopathy regressed after 1 month of steroid treatment. He was doing well when last seen.

\section{Discussion:}

The diagnosis of sarcoidosis is commonly established based on clinical and radiological findings that are supported by histological findings. The clinical signs and symptoms are nonspecific and include fatigue, general malaise, weight loss, and less commonly, fever. The characteristic radiological findings associated with sarcoidosis have been well described and the findings include bilateral hilar lymphadenopathy and parenchymal abnormalities. ${ }^{12}$ In contrast to other causes of hilar lymphadenopathy, hilar lymphadenopathy in sarcoidosis is typically bilateral and symmetric. In addition, hilar lymphadenopathy is frequently associated with mediastinal lymph node enlargement as depicted on CT scans, especially including the right paratracheal and subaortic nodes. However, mediastinal lymphadenopathy without hilar involvement is rare. ${ }^{13}$ Because of this characteristic distribution, intrathoracic lymphadenopathy typically resembles the shape of the Greek letter lambda (lambda sign). ${ }^{14}$

Some authors believe that histological proof of non-caseating granuloma is necessary to confirm the diagnosis of sarcoidosis. ${ }^{15}$ Others note that presence of BHL along with other classical features of sarcoidosis has a positive predictive value of $99.95 \%$ for diagnosis of sarcoidosis, so routine biopsy poses unnecessary risk to the patient, therefore, not advised. ${ }^{16}$ In our case, tissue diagnosis was made through CT guided FNAC of hilar lymphadenopathy which revealed the characteristics histopathology of sarcoidosis. 
Up to $35 \%$ cases of sarcoidosis are Löfgren's syndrome. ${ }^{17}$ According to Mana et al, who reported a series of 186 patients with Löfgren's syndrome, clinical features of Löfgren's syndrome included arthralgia (68\%), fever (38\%), cough or dyspnea (13\%), granulomatous skin lesion (13\%), arthritis (13\%), hepatomegaly (6\%), ocular symptoms (5\%), peripheral lymphadenopathy (4\%), splenomegaly (2\%), hypercalcemia $(2 \%){ }^{7}$ In this series, angiotensin converting enzyme was elevated in about $50 \%$ of patients. ${ }^{7}$ Our patient had arthralgia, ocular symptoms and vague respiratory symptoms. Though slit lamp examination revealed uveitis in this patient, it did not affect his visual acuity.

Most patients with Löfgren's syndrome have complete resolution of their symptoms, and this syndrome represents a benign acute presentation of sarcoidosis with a good prognosis. In the series of Löfgren, 102 patients (91.9\%) had complete resolution of hilar lymphadenopathy and infiltrates, if present, by 2 years. ${ }^{9}$ In the series of Mana et al, only 8\% of patients ( $\mathrm{n}=11)$ had active disease by 2 years of diagnosis. ${ }^{7}$ In the series of Visser et al, all patients with acute sarcoid arthritis went into clinical remission within 3 months. ${ }^{18}$ In this patient, the response to steroid was also highly satisfactory.

\section{Conclusion}

Although Löfgren's syndrome is considered as an acute form of sarcoidosis it has distinct clinical and prognostic features. It has an excellent prognosis, with higher remission or lower relapse rate than sarcoidosis. Because of presentation with joint pain, Löfgren's syndrome might be confused with rheumatoid, viral or reactive arthritis. Increased awareness among the physicians is paramount in the diagnosis and treatment of this rare but potentially curable disease.

\section{Conflict of interest: None}

\section{References}

1. Baughman RP, Lower EE, du Bois RM. Sarcoidosis. Lancet 2003; 361: 1111-1118.

2. Siltzbach LE, James DG, Neville E, et al. Course and prognosis of sarcoidosis around the world. Am J Med 1974; 57: 847-852.

3. Costabel U, Hunninghake GW. ATS/ERS/WASOG statement on sarcoidosis. Sarcoidosis Statement Committee. American Thoracic Society. Am J Respir Crit Care Med 1999; 160 (2): 736-755
4. European Respiratory Society. World Association for Sarcoidosis and Other Granulomatous Disorders. Eur Respir J 1999;14: 735-737.

5. Boeck C. Multiple benign sarcoid of the skin. J Cutan Genitourin Dis 1899; 17:543-50.

6. Heerfordt C. Uber eine Febris uveo-parotidea subchronica. Von Graefe‘s Archiv fur Opthalmologie 1909; 70:254-73. [German]

7. Mana J, Gomez-Vaquero C, Montero A, et al. Löfgren’s syndrome revisited: a study of 186 patients. Am J Med 1999; 107: 240-245.

8. Lofgren S. Primary pulmonary sarcoidosis: early signs and symptoms. Acta Med Scand 1953; 145,424-431

9. Lofgren S. Primary pulmonary sarcoidosis: clinical course and prognosis. Acta Med Scand 1953; 145,465-474

10. Rybicki BA, Major M, Popovich J Jr, et al. Racial differences in sarcoidosis incidence: a 5-year study in a health maintenance organization. Am J Epidemiol 1997; 145:234241

11. Pietinalho A, Hiraga $Y$, Hosoda $Y$ et al. The frequency of sarcoidosis in Finland and Hokkaido, Japan: a comparative epidemiological study. Sarcoidosis 1995; 12:61-67.

12. Müller NL, Kullnig P, Miller RR. The CT findings of pulmonary sarcoidosis: analysis of 25 patients. Am J Roentgenol. 1989; 152: 1179-1182.

13. Hamper UM, Fishman EK, Khouri NF et al. Typical and atypical CT manifestations of pulmonary sarcoidosis. J Comput Assist Tomogr 1986; 10:928-936.

14. Sulavik SB, Spencer RP, Weed DA et al. Recognition of distinctive patterns of gallium-67 distribution in sarcoidosis. J Nucl Med 1990;31:1909-1914

15. Tambouret R, Geisinger KR, Powers CN et al. The clinical application and cost analysis of fine-needle aspiration biopsy in the diagnosis of mass lesions in sarcoidosis. Chest 2000; 117:1004-1011

16. Reich JM, Brouns MC, O’Connor EA, et al. Mediastonoscopy in patients with presumptive stage 1 sarcoidosis a risk/benefit, cost/benefit analysis. Chest 1998; 113: $147-53$.

17. Moore AL. Löfgren's syndrome and Arthritis. J Fam Pract 1981;12:1071-72

18. Visser H, Vos K, Zanelli E, et al. Sarcoid arthritis: clinical characteristics, diagnostic aspects, and risk factors. Ann Rheum Dis 2002; 61,499-504 